\title{
Impacto del servicio profesional farmacéutico asistencial de cesación tabáquica en la farmacia comunitaria. Protocolo del estudio y características de los investigadores y los pacientes
}

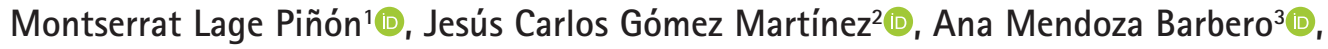
Leire Gaztelurrutia Lavesa ${ }^{4}{ }^{\oplus}$, Javier Plaza Zamora ${ }^{5}{ }^{\oplus}$, Miguel Aguiló Juanola ${ }^{6}$, Maite Climent Catalá7@i, Antonieta de Andrés Dirube ${ }^{8}{ }^{\infty}$, Luis García Moreno ${ }^{9}$, Irene Jaraiz Magariños ${ }^{10}{ }^{\circ}$, Montse Moral Ajado ${ }^{11}{ }^{\circ}$, Navidad Sánchez Marcos ${ }^{12}$ (1)

1. Grupo de Respiratorio y tabaquismo de la Sociedad Española de Farmacia Clínica, Familiar y Comunitaria. Farmacéutica comunitaria en Culleredo (A Coruña). 2. Grupo de Respiratorio y tabaquismo de la Sociedad Española de Farmacia Clínica, Familiar y Comunitaria. Farmacéutico comunitario en Barcelona (Barcelona). 3. Grupo de Respiratorio y tabaquismo de la Sociedad Española de Farmacia Clínica, Familiar y Comunitaria. Farmacéutica comunitaria en Reus (Tarragona). 4. Grupo de Respiratorio y tabaquismo de la Sociedad Española de Farmacia Clínica, Familiar y Comunitaria. Farmacéutica comunitaria en Barakaldo (Bizcaia). 5. Grupo de Respiratorio y tabaquismo de la Sociedad Española de Farmacia Clínica, Familiar y Comunitaria. Farmacéutico comunitario en Mazarrón (Murcia). 6. Grupo de Respiratorio y tabaquismo de la Sociedad Española de Farmacia Clínica, Familiar y Comunitaria. Farmacéutico comunitario en Palma (Illes Balears). 7. Farmacéutica comunitaria en L'Olleria (Valencia). 8. Farmacéutica comunitaria en Astillero (Cantabria). 9. Grupo de Respiratorio y tabaquismo de la Sociedad Española de Farmacia Clínica, Familiar y Comunitaria. Farmacéutico comunitario en Munera (Albacete). 10. Farmacéutica comunitaria en Olloniego (Asturias). 11. Grupo de Respiratorio y tabaquismo de la Sociedad Española de Farmacia Clínica, Familiar y Comunitaria. 12. Grupo de Respiratorio y tabaquismo de la Sociedad Española de Farmacia Clínica, Familiar y Comunitaria. Farmacéutica comunitaria en San Sebastián de los Reyes (Madrid).
\end{abstract}

\section{PALABRAS CLAVE}

Cesación tabáquica, farmacia comunitaria, servicio profesional farmacéutico asistencial

\section{ABREVIATURAS}

SPFA: servicio profesiona farmacéutico asistencial FC: farmacéutico comunitario CEIC: Comité Ético de Investigación Clínica

PA: presión arteria

CSRI: Client Service Receipt Inventory

IMC: índice de masa corporal

\section{RESUMEN}

Introducción: el tabaquismo es un grave problema de salud pública, el $23 \%$ de los españoles mayores de 15 años fuma a diario y el 2,4\% es fumador ocasional. El farmacéutico comunitario (FC) es un profesional sanitario accesible y cercano a la población que puede desempeñar una importante labor en cesación tabáquica. Es fundamental realizar estudios de investigación que demuestren el impacto de la intervención del FC en tabaquismo y ayuden a definir un protocolo de actuación que pueda implantarse de manera generalizada en cualquier farmacia comunitaria de nuestro pais.

Material y métodos: el protocolo se utilizó en un estudio prospectivo controlado no aleatorizado de 12 meses de seguimiento en paciente fumador mayor de 18 años que acudía a la farmacia comunitaria con una prescripción médica para dejar de fumar, haciendo una consulta para dejar de fumar o paciente que se detecta como fumador. El estudio se diseñó para desarrollarse en 100 farmacias con farmacéuticos capacitados a través del programa CESAR (grupo intervención) que realizaban una intervención estructurada y 100 farmacias con farmacéuticos no capacitados (grupo control) que realizaban una intervención habitual. Cada una de las farmacias debía reclutar un total de 5 pacientes, lo que supondría un total de 1.000 casos

Resultados: en el estudio participaron 182 FC (grupo intervención: 102, grupo control: 80), la mayoría de los cuales desarrollaban su actividad profesional en farmacias de barrio. Se incorporaron 1.078 pacientes (grupo intervención: 800, grupo control: 278), con una distribución homogénea por sexos y una edad media de 49 años.
Recibido: 28/05/2021

Aceptado: 02/08/2021

Disponible online: 11/01/2022
Financiación: el estudio fue financiado a través de una beca otorgada por el laboratorio Pfizer.

Conflicto de intereses: ninguno.

Cite este artículo como: Lage M, Gómez JC, Mendoza A, Gaztelurrutia L, Plaza J, Aguiló M, Climent M, de A, García L, Jaraiz I, Moral M, Sánchez N. Impacto del servicio profesional farmacéutico asistencial de cesación tabáquica en la farmacia comunitaria. Protocolo del estudio y características de los investigadores y los pacientes. Farmacéuticos Comunitarios. 2022 Jan 11; 14 (1): 6-15. doi:10.33620/FC.2173-9218.(2022/Vol14).001.03

Correspondencia: Montserrat Lage Piñón (mlage@sefac.org).

ISSN 1885-8619 @SEFAC (Sociedad Española de Farmacia Clínica, Familiar y Comunitaria). Todos los derechos reservados. 


\section{INTRODUCCIÓN}

El tabaquismo es un grave problema de salud pública $y$, de hecho, se calcula que el $23 \%$ de los españoles mayores de 15 años fuma a diario y el 2,4\% es fumador ocasional (1). Actualmente se considera el primer problema de salud pública prevenible en los países desarrollados. Cada año mueren en el mundo más de 8 millones de personas debido al tabaco, alrededor de 7 millones por consumo directo y 1,2 por exposición al humo del tabaco (2). En España se calcula que se producen al año más de 55.000 muertes por esta causa y siendo primera causa de muerte prematura y enfermedad prevenible (3).

La promoción de la salud es una de las actividades más importantes de la farmacia comunitaria, y el servicio profesional farmacéutico asistencial (SPFA) de cesación tabáquica se incluye dentro de estas actividades (4). Diferentes estudios han demostrado que, si este servicio se implanta en las farmacias con profesionales formados y capacitados (5), tendría como mínimo las tasas de abstinencia encontradas en estudios realizados por otros profesionales sanitarios. Además, las farmacias son establecimientos sanitarios fácilmente accesibles a la población general, por su cercanía y alto número, lo que hace que, si el servicio se implantase mayoritariamente, podría contribuir a la reducción del número de fumadores en nuestro país, con el consiguiente impacto positivo a nivel clínico, económico y humanistico.

En este sentido, se diseñó el estudio Impacto del servicio farmacéutico asistencial de cesación tabáquica en la farmacia comunitaria - Ensayo controlado no aleatorizado con seguimiento a 12 meses, cuyo objetivo principal es evaluar el impacto de un SPFA de cesación tabáquica (programa CESAR) en la farmacia comunitaria, en comparación con la intervención habitual en la mejora de la calidad de vida relacionada con la salud de pacientes fumadores que acuden a las farmacias comunitarias, así como el coste efectividad y el coste utilidad de dicho servicio, para obtener una evidencia más robusta sobre el impacto de las intervenciones de los FC en cesación tabáquica.

El objetivo concreto de este artículo es describir las caracteristicas de los FC participantes y de la muestra obtenida en el estudio, así como el protocolo seguido para su realización.

\section{MATERIAL Y MÉTODOS}

\section{Diseño del estudio}

El protocolo se llevó a cabo en un estudio prospectivo controlado no aleatorizado de 12 meses de seguimiento.

\section{Ámbito de desarrollo}

El estudio se desarrolló en farmacias comunitarias de toda España. La convocatoria para que los farmacéuticos participasen en el estudio se realizó desde SEFAC vía correo electrónico a todos los socios y fue voluntaria. Las farmacias que decidieron participar se dividieron en dos grupos, control e intervención. En el grupo intervención los farmacéuticos participantes habian sido capacitados en el servicio mediante el programa CESAR, mientras que los farmacéuticos del grupo control no habian realizado esta capacitación y ofrecian a sus pacientes la atención farmacéutica habitual.

Para cada farmacia intervención se seleccionaba una farmacia control de características similares a la farmacia intervención: tipo de farmacia (urbana, rural o turística), personal de la farmacia (número de farmacéuticos y auxiliares), caracteristicas de la farmacia (horario de apertura, dimensiones, proximidad al centro de atención primaria más cercano) y características de la población atendida (caracteristicas sociodemográficas y de nivel educativo similares).

\section{Población de estudio}

\section{Criterios de inclusión}

Fumadores mayores de 18 años que acudian a la farmacia comunitaria en alguna de las siguientes situaciones:

- Paciente con una prescripción médica para dejar de fumar.

- Paciente que hace una consulta para dejar de fumar.

- Paciente que sin haber consultado se detecta como fumador.

Se trató de una intervención proactiva, ya que no se realizó exclusivamente en pacientes fumadores que demandaban el servicio.

\section{Criterios de exclusión}

- Sujetos con deterioro cognitivo y/o enfermedad mental severo/inestable

- Sujetos con tratamiento por drogodependencias.

- Mujeres embarazadas o en periodo de lactancia.

\section{Tamaño de muestra}

El estudio se diseñó para llevarse a cabo en 100 farmacias con farmacéuticos capacitados a través del programa CESAR (grupo intervención) y 100 farmacias con farmacéuticos no capacitados (grupo control). Cada una de las farmacias debía reclutar un total de 5 pacientes, lo que supondría un total de 1.000 casos.

\section{Comité de ética}

El estudio fue evaluado favorablemente por el Comité Ético de Investigación Clínica (CEIC) de la Fundación San Juan de Dios de Barcelona en junio de 2016. 


\section{Grupo intervención}

La intervención según el programa CESAR consiste en un abordaje farmacéutico en el paciente fumador de una manera consensuada y protocolizada. La intervención consta de las siguientes fases:

\section{Captación del paciente}

Cuando el paciente acude a la farmacia con una prescripción médica de un medicamento para dejar de fumar, pide asesoramiento o se detecta después de cualquier acto farmacéutico (toma de presión arterial, [PA]), en la dispensación de un medicamento, en la indicación farmacéutica...). En el estudio se comprobó que cumplía los criterios de inclusión y se invitó al paciente a participar. En caso de que aceptara, se procedió a obtener el consentimiento informado por escrito de participación y se pasaron las escalas de evaluación en línea base. Una vez hecho esto, se ofreció cita para la entrevista inicial.

\section{Entrevista inicial}

Consiste en aquella intervención del farmacéutico con aquel paciente que está decidido a dejar de fumar y ha cumplido los requisitos anteriores. En el estudio, en la visita inicial tenían que constar los datos sociodemográficos, historia de tabaquismo, antecedentes personales y se indicaba si iniciaría tratamiento y cuál.

\section{Visitas de seguimiento}

Estas visitas están protocolizadas y se cita al paciente para cada una de ellas. El motivo de éstas es preguntar al paciente su estado a fin de poder reforzar y ayudar en aquello que tenga problemas, se pregunta sobre efectos secundarios de la medicación, adherencia al tratamiento... (en el caso que lleve tratamiento o corresponda al período que se está en tratamiento). Es el momento para que el paciente presente sus incertidumbres y se le ayude a solucionarlas. En el estudio se realizaban visitas a los 7, 15, 30, 60, 90 y 180 dias desde la visita inicial.

Visita final

Se realizó a los 12 meses.

\section{Grupo control}

Los FC control realizaban una intervención habitual en tabaquismo en su práctica profesional. Se captaba a los pacientes y se verificada que cumplían los criterios de inclusión y firmaban el consentimiento informado. Para garantizar el rigor de la investigación y mejorar el control del grupo de comparación, los FC rellenaban una ficha donde describian qué abordaje realizaban de forma habitual.

Tanto el grupo control como el de intervención en la línea base, mes 6 y mes 12 citaron a los pacientes para realizar las entrevistas de evaluación en las que se recogieron los siguientes datos:
Línea base

- Peso, PA y cooximetría.

- Sociodemográficos: sexo, edad, situación laboral, estado civil, nivel de estudios, convivencia, enfermedades crónicas.

- Grado de motivación para dejar de fumar (test de Richmond).

- Grado de dependencia a la nicotina (test de Fagerström).

- Años fumando.

- Consumo de cigarrillos/día.

- Consumo acumulado de cigarrillos ( $n^{0}$ cigarrillos días $x$ años de fumador $/ 20$ ).

Línea base, 6 y 12 meses

- PA.

- Peso.

- Cooximetría.

- Calidad de vida: medida en el cuestionario EuroQol-5D (6). Instrumento genérico de calidad de vida relacionada con la salud.

- Recaída y número de cigarrillos/día.

- Adherencia al tratamiento farmacológico (si lo hubiera) según el registro de retirada de medicación de farmacia anotado en la historia farmacoterapéutica de la farmacia.

- Uso de servicios directos e indirectos: CSRI, cuestionario de utilización de servicios sanitarios y de costes directos e indirectos.

\section{Análisis de los datos}

Todos los participantes registraron los datos recogidos en la plataforma www.investigacionsefac.org y además el grupo intervención registró las visitas en la plataforma SEFAC eXPERT (plataforma integral de gestión de SPFA, desarrollada por la Sociedad Española de Farmacia Clínica, Familiar y Comunitaria, SEFAC). El análisis de los datos obtenidos se realizó empleando el paquete estadístico STATA v16.1. La comparabilidad en línea base entre grupos se evaluó mediante test de chi-cuadrado o test exacto de Fisher para los datos categóricos y mediante test de $t$ de Student para las variables continuas. Para evaluar la efectividad de la intervención CESAR se utilizarán modelos multivariantes lineales y logísticos para las variables continuas y dicotómicas, respectivamente.

\section{RESULTADOS}

\section{Características sociodemográficas de los farmacéuticos}

En el estudio, cuyas fases están esquematizadas en la figura 1, participaron a nivel nacional un total de $182 \mathrm{FC}$ ) de los cuales 102 pertenecian al grupo intervención y 80 al grupo control (figura 2). La mayoría era mujer $(68,1 \%)$. 


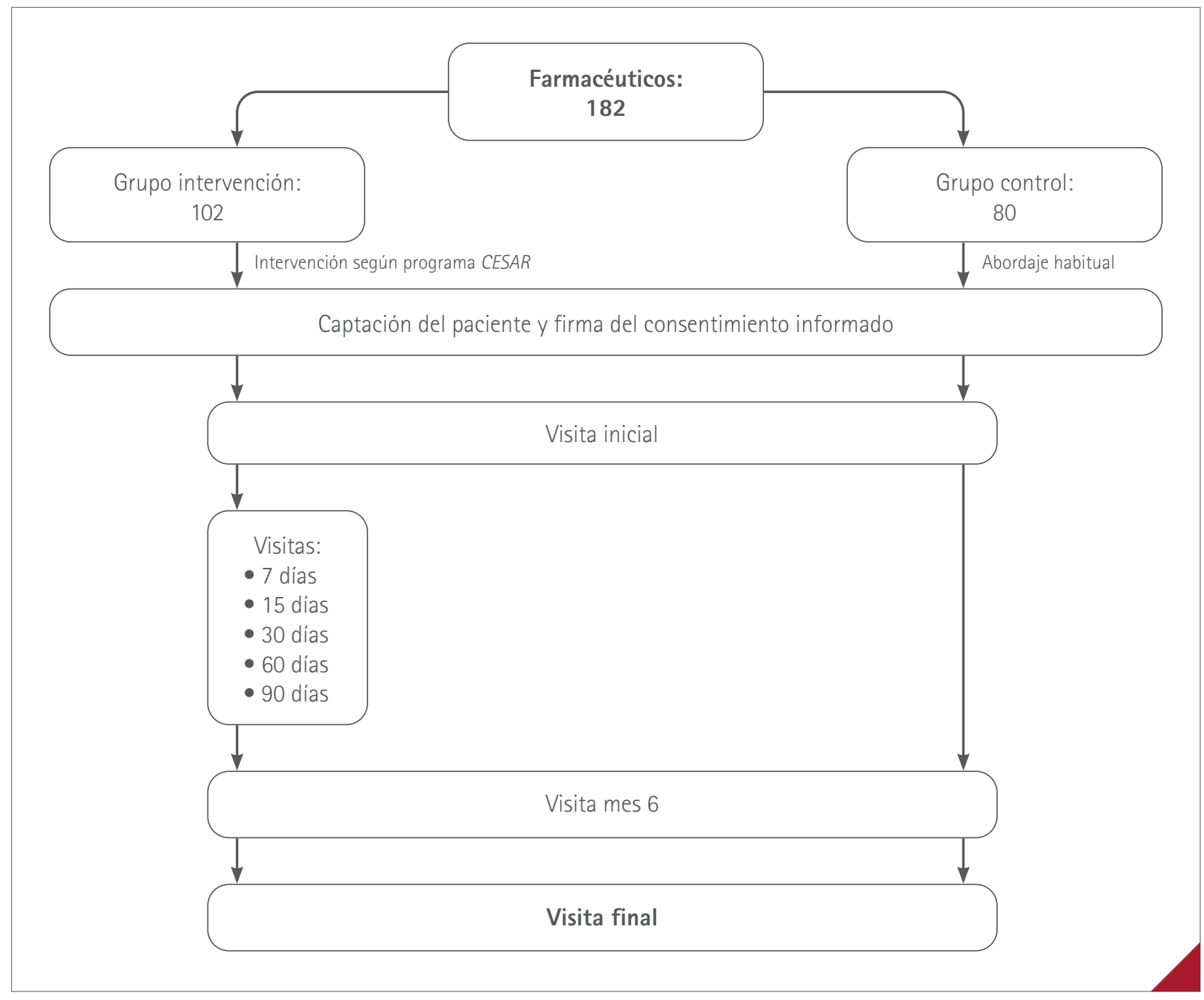

Figura 1 FC participantes en el estudio y visitas realizadas en cada uno de los grupos

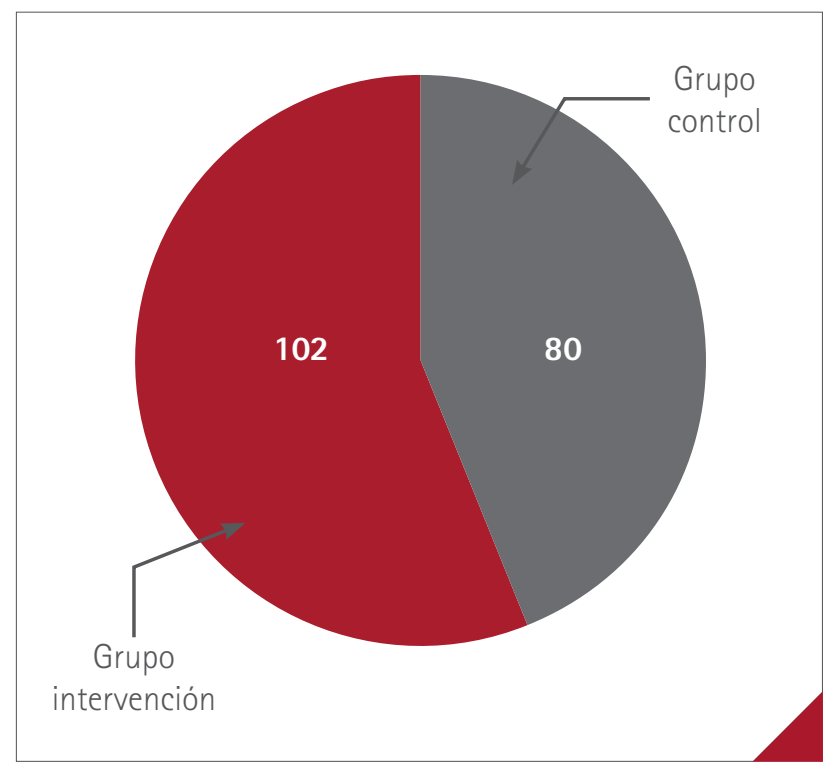

Figura 2 FC participantes en el grupo control y en el grupo intervención
En el tipo de FC que participó en el estudio (titular, sustituto o adjunto) se encontraron diferencias estadisticamente significativas, siendo la proporción de titulares superior en el grupo control $(63,8 \%)$, mientras que en el grupo intervención hubo una mayor proporción de adjuntos (50\%). En cuanto al tipo de farmacia participante (turística, de paso, de barrio, cercana a un centro de salud o a un centro comercial), la mayoría fueron farmacias de barrio (75\% en el grupo control y $66,7 \%$ en el grupo intervención). Entre grupos no hubo diferencias significativas $p=0,158$.

Además, se detectaron diferencias estadísticamente significativas entre grupos en el tamaño del municipio de referencia y en número de farmacéuticos titulares, adjuntos y técnicos/auxiliares en las farmacias. En las farmacias del grupo control era más frecuente que hubiera un mayor número de titulares; por el contrario, el número de adjuntos y auxiliares era superior en las farmacias del grupo intervención.

La tabla 1 muestra las características sociodemográficas de los FC participantes en el estudio. 
Tabla 1 Características sociodemográficas de los FC participantes en el estudio

\begin{tabular}{|c|c|c|c|}
\hline Variable & Grupo control $(n=80)$ & Grupo CESAR $(\mathrm{n}=102)$ & p-valor* \\
\hline Sexo n (\%) & & & 0,424 \\
\hline Hombre & $23(28,8)$ & $35(34,4)$ & \\
\hline Mujer & $57(71,13)$ & $67(65,7)$ & \\
\hline \multicolumn{4}{|l|}{ Puesto que desempeña, n (\%) } \\
\hline Farmacéutico titular & $51(63,8)$ & $41(40,2)$ & Ref. \\
\hline Farmacéutico sustituto & $4(5,0)$ & $10(9,8)$ & 0,071 \\
\hline Farmacéutico adjunto & $25(31,3)$ & $51(50,0)$ & 0,004 \\
\hline Tipo de farmacia, n (\%) & & & 0,158 \\
\hline Farmacia turistica & $7(8,8)$ & $4(3,9)$ & Ref. \\
\hline Farmacia de paso & $6(7,5)$ & $9(8,8)$ & 0,239 \\
\hline Farmacia de barrio & $60(75,0)$ & $68(66,7)$ & 0,293 \\
\hline Farmacia cercana a un centro de salud & $7(8,8)$ & $20(19,6)$ & 0,035 \\
\hline Farmacia de centro comercial & $0(0,0)$ & $1(1,0)$ & \\
\hline \multicolumn{4}{|l|}{ Tamaño municipio farmacia (habitantes), n (\%) } \\
\hline$<10.000$ & $32(40,0)$ & $31(30,4)$ & Ref. \\
\hline $10.000-50.000$ & $15(18,8)$ & $33(32,4)$ & 0,041 \\
\hline $50.000-500.000$ & $18(22,5)$ & $31(30,40)$ & 0,139 \\
\hline$>500.000$ & $15(18,8)$ & $7(6,9)$ & 0,162 \\
\hline \multicolumn{2}{|l|}{ Número de farmacéuticos titulares, n (\%) } & & 0,033 \\
\hline 1 & $60(75,0)$ & $89(87,3)$ & \\
\hline$>1$ & $20(25,0)$ & $13(12,7)$ & \\
\hline Número de farmacéuticos adjuntos, media (DE) & $1,4(1,2)$ & $2,3(1,8)$ & 0,000 \\
\hline Número de técnicos auxiliares, media (DE) & $1,9(1,0)$ & $2,5(2,4)$ & 0,020 \\
\hline
\end{tabular}

* p-valor según modelos de regresión logística.

Las diferencias estadisticamente significativas están marcadas con un recuadro rojo.

\section{Características sociodemográficas y clínicas de los pacientes}

Se incorporaron un total de 1.078 pacientes al estudio, 800 asignados al grupo intervención y 278 al grupo control (figura 3). La proporción de hombres y mujeres entre grupos fue similar (grupo intervención: $57 \%$ mujeres / $43 \%$ hombres, grupo control: 55,8\% mujeres /44,2\% hombres) y la edad media se situó en torno a los 49 años. La mayoría de los pacientes estaban casados, vivían acompañados, tenían estudios secundarios o superiores y estaban en activo. El indice de masa corporal (IMC) medio fue de $26 \mathrm{~kg} / \mathrm{m}^{2}$ y las enfermedades más frecuentes que padecian fueron la hipertensión arterial, dislipemias, y enfermedades respiratorias.

Se detectaron diferencias estadisticamente significativas entre los participantes del grupo control y el grupo intervención en el nivel de estudios, con una mayor proporción de personas con estudios superiores en el primero $(34,9 \%$ vs. $25,9 \% p=0,046)$. También se

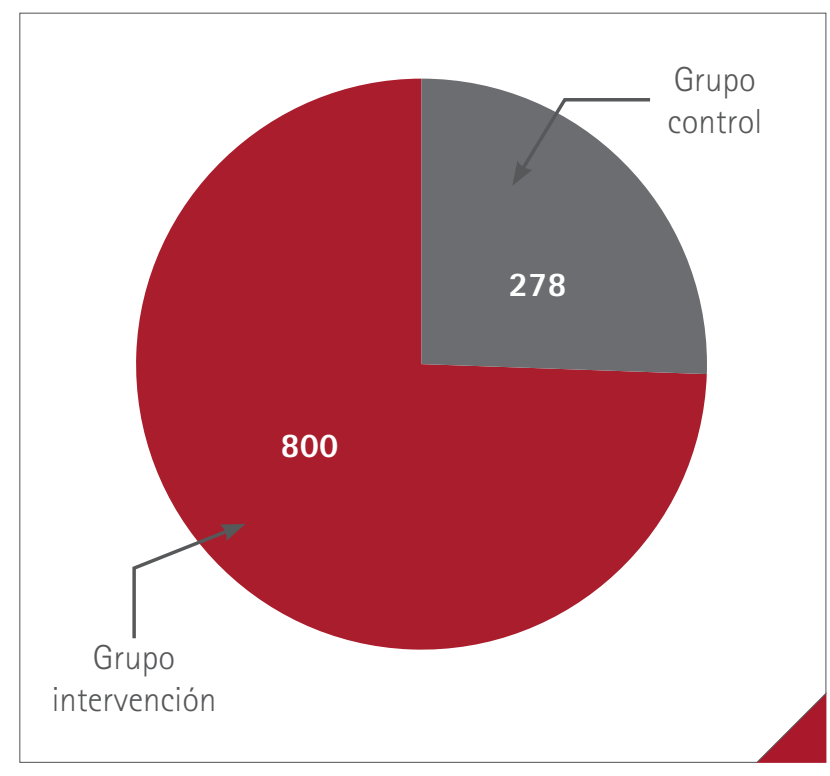

Figura 3 Pacientes fumadores en el grupo control y en el grupo intervención 
observaron diferencias en el número de enfermedades $(p=0,018)$, siendo superior en el control $(1,3$ vs. 1,03$)$, la presencia de hipertensión arterial también fue significativamente $(p=0,007)$ más frecuente $(19,1 \%$ vs. $12,5 \%)$ en este grupo. La calidad de vida relacionada con la salud en la línea base fue significativamente $(p=0,013)$ superior en el grupo intervención $(0,84$ vs. 0,81) (en el Euro0ol 5D el resultado de la medición oscila entre 0 y 1 , donde 0 es la muerte y 1 el mejor estado de salud posible).

La tabla 2 muestra las características sociodemográficas y clínicas de los pacientes incluidos en el estudio.

Tabla 2 Datos sociodemográficos y clínicos de los pacientes incluidos en el estudio en la línea base

\begin{tabular}{|c|c|c|c|c|c|}
\hline Variable & $\begin{array}{l}\text { Grupo control } \\
\qquad N=278\end{array}$ & $\% m$ & $\begin{array}{l}\text { Grupo CESAR } \\
\qquad \mathrm{N}=800\end{array}$ & $\% m$ & p-valor* \\
\hline Género, n (\%) & & 0 & & 0 & 0,718 \\
\hline Mujer & $155(55,8)$ & & $456(57,0)$ & & \\
\hline Hombre & $123(44,2)$ & & $344(43,0)$ & & \\
\hline Edad, media (DE) & $49,7(12,3)$ & 0 & $48,4(11,8)$ & 0 & 0,116 \\
\hline Estado civil, n (\%) & & 1,8 & & 2,5 & \\
\hline Casado/a o emparejado & $168(60,4)$ & & $510(63,8)$ & & Ref. \\
\hline Separado/a o divorciado & $29(10,4)$ & & $108(13,5)$ & & 0,393 \\
\hline Soltero/a & $69(24,8)$ & & $133(16,6)$ & & 0,011 \\
\hline Viudo/a & $7(2,5)$ & & $29(3,6)$ & & 0,465 \\
\hline Convivencia, n (\%) & & 1,1 & & 2,5 & 0,374 \\
\hline Vive acompañado & $230(82,7)$ & & $671(83,9)$ & & \\
\hline Vive solo & $45(16,2)$ & & $109(13,6)$ & & \\
\hline Nivel de estudios, n (\%) & & 1,8 & & 3,8 & \\
\hline Sin estudios & $5(1,8)$ & & $11(1,4)$ & & 0,462 \\
\hline Graduado escolar & $43(15,5)$ & & $115(14,4)$ & & 0,346 \\
\hline Estudios primarios & $37(13,3)$ & & $125(15,6)$ & & Ref. \\
\hline Estudios secundarios & $91(32,7)$ & & $312(39,0)$ & & 0,976 \\
\hline Estudios superiores & $97(34,9)$ & & $207(25,9)$ & & 0,043 \\
\hline Situación laboral, n (\%) & & 0 & & 1,0 & \\
\hline Amo/a de casa & $26(9,4)$ & & $73(9,1)$ & & Ref. \\
\hline Desempleado con subsidio & $16(5,8)$ & & $52(6,5)$ & & 0,687 \\
\hline Desempleado sin subsidio & $8(2,9)$ & & $21(2,6)$ & & 0,889 \\
\hline Empleado/a con baja laboral & $10(3,6)$ & & $23(2,9)$ & & 0,658 \\
\hline En activo & $172(61,9)$ & & $510(63,8)$ & & 0,816 \\
\hline Estudiante & $4(1,4)$ & & $14(1,8)$ & & 0,720 \\
\hline Invalidez laboral transitoria & $2(0,7)$ & & $5(0,6)$ & & 0,894 \\
\hline Jubilado/a & $40(14,4)$ & & $94(11,8)$ & & 0,552 \\
\hline IMC $\left(\mathrm{kg} / \mathrm{m}^{2}\right)$, media (DE) & $26,1(4,5)$ & 3,6 & $26,2(4,7)$ & 8,6 & 0,868 \\
\hline Número de enfermedades & $1,3(1,7)$ & 0 & $1,03(1,5)$ & 0 & 0,018 \\
\hline
\end{tabular}

\%: porcentaje de sujetos que no tienen registrada la información de la variable.

*p-valor según modelos de regresión logística realizados sobre la base de datos imputada.

Las diferencias estadisticamente significativas están mercadas con un recuadro rojo. 
Tabla 2 (Continuación) Datos sociodemográficos y clínicos de los pacientes incluidos en el estudio en la línea base

\begin{tabular}{|c|c|c|c|c|c|}
\hline Variable & $\begin{array}{l}\text { Grupo control } \\
\qquad=278\end{array}$ & $\% m$ & $\begin{array}{c}\text { Grupo CESAR } \\
\quad \mathrm{N}=800\end{array}$ & $\% m$ & p-valor* \\
\hline Comorbilidades, n (\%) & & 0 & & 0 & \\
\hline HTA & $53(19,1)$ & & $100(12,5)$ & & 0,007 \\
\hline Colesterol & $48(17,3)$ & & $106(13,3)$ & & 0,100 \\
\hline Diabetes & $22(7,9)$ & & $40(5,0)$ & & 0,075 \\
\hline Neoplasias & $5(1,8)$ & & $13(1,6)$ & & 0,846 \\
\hline Enfermedad renal & $3(1,1)$ & & $7(0,9)$ & & 0,760 \\
\hline Enfermedad hepática & $1(0,4)$ & & $7(0,9)$ & & 0,404 \\
\hline Enfermedad neurológica & $7(2,5)$ & & $20(2,5)$ & & 0,987 \\
\hline Enfermedad endocrina & $14(5,0)$ & & $41(5,1)$ & & 0,954 \\
\hline Enfermedad dermatológica & $4(1,1)$ & & $28(3,5)$ & & 0,091 \\
\hline Enfermedad mental leve o enfermedad mental crónica estable & $23(8,3)$ & & $54(6,8)$ & & 0,396 \\
\hline Enfermedad músculo esquelética & $21(7,6)$ & & $54(6,8)$ & & 0,650 \\
\hline Enfermedad respiratoria & $29(10,4)$ & & $85(10,6)$ & & 0,928 \\
\hline Cardiopatía & $14(5,0)$ & & $28(3,5)$ & & 0,257 \\
\hline Trastornos del sueño & $22(7,9)$ & & $43(5,4)$ & & 0,128 \\
\hline Trastornos digestivos & $19(6,8)$ & & $45(5,6)$ & & 0,463 \\
\hline Problemas oftalmológicos & $9(3,2)$ & & $19(2,4)$ & & 0,438 \\
\hline Problemas odontológicos & $10(3,6)$ & & $15(1,9)$ & & 0,106 \\
\hline Alcohol y otras drogas & $2(0,7)$ & & $7(0,9)$ & & 0,806 \\
\hline Alergias a medicamentos, alimentos u otras alergias & $51(18,4)$ & & $110(13,8)$ & & 0,065 \\
\hline EQ-5D-3L, media (DE) & $0,81(0,20)$ & 0,7 & $0,84(0,18)$ & 0,4 & 0,013 \\
\hline
\end{tabular}

\%: porcentaje de sujetos que no tienen registrada la información de la variable.

*p-valor según modelos de regresión logística realizados sobre la base de datos imputada.

Las diferencias estadisticamente significativas están mercadas con un recuadro rojo.

\section{DISCUSIÓN}

Este estudio controlado no aleatorizado sobre el impacto de la intervención de los FC en cesación tabáquica es el primero de estas caracteristicas realizado en nuestro pais y uno de los más completos a nivel internacional, ya que no solo evalúa la efectividad de dicha intervención, sino que además nos permitirá conocer su impacto en la calidad de vida de los pacientes, así como si se trata de un servicio coste-efectivo.

Una de las caracteristicas más importantes es el tamaño de muestra alcanzada, tanto de farmacéuticos (182) como de pacientes (1.078). En la tabla 3 se pueden observar los tamaños de muestra con los que se trabajó en otros estudios realizados en farmacia comunitaria ya publicados, siendo estos inferiores.

Otro aspecto diferencial, fue el hecho de que la captación de los pacientes se realizó de manera proactiva, ya que no solo se ofertaba el servicio a sujetos que lo demandasen, sino que también se ofertaba a todos aquellos que el farmacéutico detectaba como fumadores y a aquellos que acudian a la farmacia con una prescripción médica para dejar de fumar.

Esto también ha podido influir en el elevado tamaño de muestra conseguido.

En la tabla 4 se puede observar cómo se realizó la captación de los pacientes, siguiendo diferentes criterios, en distintos estudios llevados a cabo en farmacia comunitaria.

La intervención realizada en este estudio ha sido estructurada e intensiva. Existe una revisión Cochrane (5), en la que los autores concluyen que probablemente la atención más intensiva en la farmacia comunitaria ayude a las personas a dejar de fumar más que las intervenciones menos intensivas (tabla 5). 
Tabla 3 FC y pacientes participantes en diferentes estudios de cesación tabáquica realizados en la farmacia comunitaria

\begin{tabular}{|c|c|c|}
\hline Estudio & № fumadores incluidos & FC participantes \\
\hline $\begin{array}{l}\text { Training pharmacists and pharmacy assistants in the stage-of-change } \\
\text { model of smoking cessation: a randomised controlled trial in Scotland. } \\
\text { Sinclair et al. (7) (1998) }\end{array}$ & $\begin{array}{l}\text { 492: } \\
\text { - } 224 \text { grupo intervención } \\
\text { - } 268 \text { grupo control }\end{array}$ & $\begin{array}{l}\text { - } 40 \text { farmacéuticos } \\
\text { - } 54 \text { técnicos }\end{array}$ \\
\hline $\begin{array}{l}\text { A randomized controlled trial of a smoking cessation intervention } \\
\text { based in community pharmacies. } \\
\text { Maguire TA et al. (8) (2001) }\end{array}$ & $\begin{array}{l}\text { 484: } \\
\text { - } 265 \text { grupo intervención } \\
\text { - } 219 \text { grupo control }\end{array}$ & - 124 farmacéuticos \\
\hline $\begin{array}{l}\text { Deshabituación tabáquica desde la farmacia comunitaria. } \\
\text { Barbero et al. (9) (2007) }\end{array}$ & 77 & $\begin{array}{l}\text { No especificado } \\
\text { (participaron } 2 \\
\text { farmacias) }\end{array}$ \\
\hline $\begin{array}{l}\text { Effectiveness of a pharmacist-delivered smoking cessation program in } \\
\text { the State of Qatar: a randomized controlled trial. } \\
\text { El Hajj MS et al. (10) (2017) }\end{array}$ & $\begin{array}{l}\text { 314: } \\
\text { - } 167 \text { grupo intervención } \\
\text { - } 147 \text { grupo control }\end{array}$ & - 16 farmacéuticos \\
\hline
\end{tabular}

Tabla 4 Tipos de población diana elegida en estudios de cesación tabáquica en farmacia comunitaria

\begin{tabular}{|c|c|c|}
\hline Estudio & Población diana & $\begin{array}{l}\text { No de fumadores } \\
\text { incluidos }\end{array}$ \\
\hline $\begin{array}{l}\text { Training pharmacists and pharmacy assistants in } \\
\text { the stage-of-change model of smoking cessation: a } \\
\text { randomised controlled trial in Scotland. } \\
\text { Sinclair et al. (7) (1998) }\end{array}$ & $\begin{array}{l}\text { Sujetos que acuden a la FC solicitando } \\
\text { asesoramiento para dejar de fumar o un } \\
\text { producto con esta misma finalidad }\end{array}$ & 492 \\
\hline $\begin{array}{l}\text { A randomized controlled trial of a smoking cessation } \\
\text { intervention based in community pharmacies. } \\
\text { Maguire TA et al. (8) (2001) }\end{array}$ & $\begin{array}{l}\text { Sujetos que acuden a la FC y expresan su } \\
\text { deseo de dejar de fumar }\end{array}$ & 484 \\
\hline $\begin{array}{l}\text { Deshabituación tabáquica desde la farmacia } \\
\text { comunitaria. } \\
\text { Barbero et al. (9) (2007) }\end{array}$ & $\begin{array}{l}\text { Sujetos que libremente solicitan participar en el } \\
\text { programa de cesación tabáquica publicitado en } \\
\text { las farmacias participantes mediante cartelería }\end{array}$ & 77 \\
\hline $\begin{array}{l}\text { Randomized trial assessing the effectiveness of a } \\
\text { pharmacist-delivered program for smoking cessation. } \\
\text { Dent el al. (11) (2009) }\end{array}$ & Sujetos motivados para dejar de fumar & 101 \\
\hline $\begin{array}{l}\text { A mixed methods feasibility study of nicotine- } \\
\text { assisted smoking reduction programmes delivered by } \\
\text { community pharmacists - The RedPharm study. } \\
\text { Farley et al. (12) (2017) }\end{array}$ & $\begin{array}{l}\text { Sujetos que no querian dejar de fumar en } \\
\text { las próximas } 4 \text { semanas, pero que querian } \\
\text { disminuir su consumo }\end{array}$ & 68 \\
\hline
\end{tabular}

Tabla 5 Resultados de intervención en estudios realizados por FC en cesación tabáquica a 6 meses

\begin{tabular}{|l|l|c|}
\hline Estudio & Tipo de intervención & $\begin{array}{c}\% \text { cesación } \\
6 \text { meses }\end{array}$ \\
\hline $\begin{array}{l}\text { Training pharmacists and pharmacy assistants in } \\
\text { the stage-of-change model of smoking cessation: a } \\
\text { randomised controlled trial in Scotland. } \\
\text { Sinclair et al. (7) (1998) }\end{array}$ & $\begin{array}{l}\text { Breve (asesoramiento inicial y seguimiento al } \\
\text { primer, cuarto y noveno mes) }\end{array}$ & 11,6 \\
\hline $\begin{array}{l}\text { A randomized controlled trial of a smoking cessation } \\
\text { intervention based in community pharmacies. }\end{array}$ & $\begin{array}{l}\text { Estructurada (asesoramiento estructurado semanal } \\
\text { por } 4 \text { semanas y posteriormente cada mes según } \\
\text { necesidad) }\end{array}$ & 14,3 \\
\hline $\begin{array}{l}\text { Randomire TA et al. (8) (2001) } \\
\text { pharmacist-delivered program for smoking cessation. } \\
\text { Dent el al. (11) (2009) }\end{array}$ & $\begin{array}{l}\text { Grupo intervención: 3 sesiones cara a cara con el FC } \\
\text { realizada por el FC a través del teléfono }\end{array}$ & 28 \\
\hline $\begin{array}{l}\text { Effectiveness of a pharmacist-delivered smoking cessation } \\
\text { program in the State of Oatar: a randomized controlled trial } \\
\text { El Hajj MS et al. (10) (2017) }\end{array}$ & $\begin{array}{l}\text { Estructurada (con } 4 \text { sesiones en total espaciadas de } \\
2 \text { a } 4 \text { semanas) }\end{array}$ & 11,8 \\
\hline
\end{tabular}


Entre las limitaciones del estudio se encuentra la falta de aleatorización de los participantes entre los grupos control e intervención, lo que ha podido sesgar los resultados obtenidos por no garantizarse la homogeneidad entre ambos grupos. Otra de las limitaciones del estudio es la gran diferencia entre el tamaño del grupo intervención (800 fumadores) y el del grupo control (278 fumadores), lo que dificulta su comparabilidad. Otro factor que puede afectar a los resultados es el hecho de que las farmacias se autoseleccionaban, siendo posible que las del grupo intervención estuviesen más motivadas y pudiendo esto hacer que sus intervenciones fuesen más numerosas e intensas y, por tanto, con una mayor tasa de éxito.

\section{AGRADECIMIENTOS}

A todos los farmacéuticos comunitarios que han participado en el estudio por su implicación: Adela Martín Oliveros, Alejandro Eguilleor Villena, Alfonso López Ruiz, Almudena Iglesias Maceiras, Amalia García Delgado, Ana Cárdeno Galván, Ana García Pineda, Ana Mateos Lardiés, Ana Mendoza Barbero, Ana Molinero Crespo, Ana Pérez Vizcayno, Andrea Úbeda Tomás, Ángela Hernández González, Anna Berenguer Lerma, Anna Busquets i Casso, Anna Montagut Calvo, Antonia Fornaris Ramis, Antonieta de Andrés Dirube, Antonio Veciana Nogués, Araceli Díez Huidobro, Assumpta Carreras Garriga, Beatriz Sánchez Rubio, Begoña Rodríguez Fernández, Blanca Monllor Córcoles, Carlos Engel Cellier, Carmen Baldonedo Mosteiro, Carmen Cortiella Garreta, Carmen Vinagre Sánchez, Clara Ferrer Ruiz, Concepción García Vivancos, Daniel García Lerma, Dora Sivera Signes, Eduardo Agustín Álvarez, Eduardo Satué de Velasco, Elena Gimeno Navarro, Eloi Merencio Naudin, Enrique Pelleró Amorós, Esperanza Muñoz Muñoz, Eugenia García Zaragoza, Eugenia López Gómez, Felipe Alonso Peñil Peñil, Fernando Mud Castelló, Fernando Rodríguez Garfía, Francisca Ruiz Lozano, Francisco Javier Dieguez Couso, Francisco Javier Teruel Fernández, Francisco Sánchez Luengo, Francisco Torres Neira, Gema Calderón Fraile, Germán López Pazo, Gorka Areitio Laorden, Inmaculada Ruiz Alarma, İ̃̃igo Peunte Mendizabal, Irene Jaraiz Magariños, Isabel Maria Ramírez Rodríguez, Isidoro Souto Bethencourt, Itxasne GabiIondo Zelaia, Izaskun García Sarasola, Jaime Ortega Meder, Javier Boleda Relats, Javier Plaza Zamora, Javier Zaro Rosado, Jesús Carlos Gómez Martínez, Joan Lluis Palmer Llanares, Joaquín Franco Madera, Joaquín Ríos Rodríguez, Joaquín Tormo Marti, Joaquina Huarte Royo, Jorge Granados Florez, José Enrique Fuentes de Frutos, Jose Jerónimo García García, Juan Uriarte García-Borreguero, Juana Frau Bonafé, Laia Matarranz Torres, Laura Sánchez Palau, Leire Gaztelurrutia Lavesa, Liana Alemán Rangel, Lina Granell Vidal, Lourdes Pérez Mora, Lourdes Romero Toledo, Lucía García Recarey, Luis Alfonso Brizuela Rodicio, Luis García Moreno, Luis Montalbán Soler, Luis Salar Ibáñez, Luis Hernández Tomás, María
Amparo Pérez Benajas, María Carmen Chaparro Torres, María Do Carmen Raíces Madriñán, María Esther Solera Serra, Manuel Castillo López, Mara Ferrer Maestre, María Ángeles Ballester, María Ángeles Ruiz Vázquez, María Ángles Polanco Abad, María Auxiliadora Naranjo Caro, María del Mar Sole Pons, María del Mar Velert Vila, María Dolores Fernández Otero, María Dolores Díaz Díez, María Echevarría Casillas, María Edelmira Córcoles Ferrandiz, María Fernández Casado, María Guadalupe González Pérez, María Isabel Clavería Cintora, María Jesusa González Escanilla, María José Cachafeiro Jardón, María José de la Matta Martín, María José Fiz Valero, María José Matovelle Gómez, María José Mora Luque, María José Muniesa Marín, Marina Torrecillas Díaz, Marta Burniol García, Marta Gisbert Garzarán, Martín Ramírez de Diego, Matilde Beneto Borja, Mercedes Arias Puente, Mercedes Cusí Benassar, Mercedes Torres Pérez, Miguel Aguiló Juanola, Miguel Cano Ivorra, Miguel de la Fuente Martínez, Modesta Gil García, Montserrat Compte Ramisa, Montserrat Lage Piñón, Nadia Alcaraz Fernández, Navidad Sánchez Marcos, Néstor Sánchez Posada, Neus Caelles Franch, Noa Rey Torres, Noelia Tejedor García, Nuria Barrera Pujol, Núria Pérez López, Nuria Ribeiro Lanza, Nuria Solé Garrido, Nuria Valeira Pereira, Olga Deleito Molina, Olga María Piñeiro Galgo, Pablo Mora Ortiz, Patricia Alonso Lovera, Patricia Menéndez Martínez, Paula Arrandiaga Ellauri, Pedro Gabriel Gil Espallardo, Pepa Cortés Botella, Pilar Ávila Martín, Pilar Buenavida Jurado, Pilar Samper Casafranca, Rafael Lago Leonardo, Rafael Matas Aguilera, Raquel Camporro Álvarez, Raquel Garcia Fuentes, Raquel San Martín Ursa, Rita de la Plaza Zubizarreta, Roi Cal Seijas, Ricard Casas Jansá, Rosa Llul Vila, Rosa Prats Más, Rosario Mena Escobar, Rubén Sánchez Martorell, Salvador Tous Trepat, Sandra Espiño Barcala, Sara Bellver Beltrán, Sara Marín Boloquio, Sara Mud Castello, Sergio Aznar Gavilán, Soledad Maldonado Barach, Sonia Antich Artqué, Sonia Cebrián Fajardo, Tomás Sástre Báuza, Vanesa Fernández García, Vanessa Piera Villora, Yolanda Forcadell Berenguer, Yolanda García Montaña, Yolanda Herrero González, Zeneida Perseguer, Pilar Fluxa Cañellas, Gema Domínguez Urbistondo.

\section{REFERENCIAS BIBLIOGRÁFICAS}

1. Ministerio de Sanidad, Servicios Sociales e Igualdad. Hábitos de vida. Informe Anual del Sistema Nacional de Salud 2016 [Internet]. 2017 [citado 15 abril 2021]. Disponible en: https://www.mscbs.gob.es/estadEstudios/estadisticas/sisInfSanSNS/tablasEstadisticas/InfAnualSNS2016/2Hab_vida.pdf

2. Organización Mundial de la Salud. Tabaco. Datos y cifras [Internet]. 2019 [citado 15 abril 2021]. Disponible en: https://www.who.int/es/ news-room/fact-sheets/detail/tobacco

3. Organización Médica Colegial de España. Enfermedad Pulmonar Obstructiva Crónica. [Internet]. 2003 [citado 15 abril 2021]. Disponible en: https://www.cgcom.es/sites/default/files/09_tabaquismo_y_epoc_0.pdf

4. Foro de Atención Farmacéutica-Farmacia Comunitaria. Guía práctica para los Servicios Profesionales Farmacéuticos Asistenciales en la Farmacia Comunitaria [Internet]. Madrid. Consejo General de Colegios Oficiales de Farmacéuticos; 2019 [citado 15 abril 2021]. 
Disponible en: https://www.sefac.org/system/files/2021-02/AF_GUIA_ SPFA_FORO_2021_ONLINE_PGs.pdf

5. Carson-Chahhoud KV, Livingstone-Banks J, Sharrad KJ, Kopsaftis Z, Brinn MP, To-A-Nan R, et al. Community pharmacy personnel interventions for smoking cessation. Cochrane Tobacco Addiction Group, editor. Cochrane Database of Systematic Reviews [Internet]. 2019 [citado 15 abril 2021]. doi:10.1002/14651858.CD003698.pub3

6. Herdman M., Badia X., Berra S. El Euro0ol-5D: Una alternativa sencilla para la medición de la calidad de vida relacionada con la salud en atención primaria [citado 10 diciembre 2021]; Aten. Primaria. 2001 28:425-429. doi:10.1016/S0212-6567(01)70406-4.

7. Sinclair HK, Bond CM, Lennox AS, Silcock J, Winfield AJ, Donnan PT. Training pharmacist and pharmacy assistants in the stage-of-change model of smoking cessation: a randomised controIled trial in Scotland. Tob control [Internet]. 1998 [citado 15 abril 2021]; 7(3):253-261. Disponible en: https://pubmed.ncbi.nlm.nih. gov/9825420/

8. Maguire TA, McElnay JC, Drummond A. A randomized controlled trial of a smoking cessation intervention based in community pharmacies. Addiction [Internet]. 2001 [citado 15 abril 2021]; 96(2):325-331. Disponible en: https://pubmed.ncbi.nlm.nih.gov/11182878/
9. Barbero González JA, Quintas Rodríguez AM, Camacho JE. Deshabituación tabáquica desde la farmacia comunitaria. Aten Primaria [Internet]. 2000 [citado 15 abril 2021]; 26(10):693-696. Disponible en: https://www.elsevier.es/es-revista-atencion-primaria-27-articulo-deshabituacion-tabaquica-desde-farmacia-comunitaria-S0212656700787543

10. El Hajj MS, Kheir N, Al Mulla AM, Shami R, Fanous N, Mahfoud ZR. Effectiveness of a pharmacist-delivered smoking cessation program in the State of Qatar: a randomized controlled trial. BMC Public Health [Internet]. 2017 [citado 15 abril 2021]; 17(1):215. doi:10.1186/ s12889-017-4103-4

11. Dent LA, Harris KJ, Noonan CW. Randomized trial assessing the effectiveness of a pharmacist-delivered program for smoking cessation. Ann Pharmacother [Internet]. 2009 [citado 15 abril 2021]; 43(2):194-201. doi:10.1345\%2Faph.1L556

12. Farley A, Tearne S, Taskila T, Williams RH, MacAskill S, Etter J-F et al. A mixed methods feasibility study of nicotine-assisted smoking reduction programmes delivered by community pharmacists - The RedPharm study. BMC Public Health [Internet]. 2017 [citado 15 abril 2021]; 210. Disponible en: https://bmcpublichealth.biomedcentral. com/articles/10.1186/s12889-017-4116-z 